\title{
A review of gambling studies with a singaporean clinical population from 2009 to 2013
}

\begin{abstract}
This paper does a separate and independent review of the various studies put forth by National Addictions Management Service (NAMS) in relation to pathological gambling from 2009 to 2013 . The findings are analyzed in totality and split into 2 sections, looking at the evolving demographics of help-seeking gamblers (looking at a clinical population as opposed to general public targeted by the NCPG survey) over the years and the predictors of treatment outcomes. It was found that, over the years, (i) more gamblers are driven to gamble as a way to cope with their psychiatric co-morbidities and (ii) psychologist distress is also a common outcome due to the consequences of gambling. It was also found that there is a need to further examine the standard psychosocial treatment guidelines (mainly based on a cognitive behavioural approach) for the gamblers to better meet the idiosyncratic needs of the non-strategic gambler.
\end{abstract}

Keywords: pathological gambling, demographics, clinical population, psychological distress
Volume 3 Issue 4 - 2017

Lawrence Tan, Guo Song, Cheok Cheng Soon
Institute of Mental Health, Singapore

Correspondence: Lawrence Tan, Institute of Mental Health, Buangkok Green Medical Park, I 0 BuangkokView, S (539747), Singapore, Tel +65964673 I 3, Fax +6563892388 ,

Email Kok_Kah_Tan@imh.com.sg

Received: April 26, 2017| Published: May 05, 2017
Abbreviations: NCPG, national council of problem gambling; NAMS, national addictions management service; GSAS, gambling symptom assessment scale; PWI, personal wellbeing index

\section{Introduction}

When Singapore first introduced the two integrated resorts in 2010 offering casino gambling as one of its major attraction, public concerns were raised about the negative social impact of casino gambling, citing worries that the casinos could encourage more gambling and increase the risk of compulsive gambling. ${ }^{1}$ Activist groups have also argued that a casino could also lead to undesirable activities often associated with gambling, including money laundering, loan sharks or even organized crime. ${ }^{2}$ In addition to setting up social safeguards like the various types of casino exclusion/limitations that could prevent or limit casino accessibility to citizens and permanent residents, the National Council of problem gambling (NCPG) has conducted public surveys on participation of gambling activities among Singapore residents on 2005, 2008, 2011 and 2014 respectively. Of particular interest is the comparison of the prevalence rate of probable problem and pathological gamblers between 2008 and 2011 (to examine if there is a significant increase after the casinos are in operating) \& between 2011 and 2015 (after the casinos have been in place for $4-5$ years). The findings indicated a slight decrease in the prevalence rate from 2008 to 2011 (2.9\% to $2.6 \%)$ and another drastic dip from 2011 to 2014 $(2.6 \%$ to $0.7 \%)$. This paper does a separate and independent review of the various studies put forth by National Addictions Management Service (NAMS) in relation to pathological gambling from 2009 to 2013. The findings are analyzed in totality and split into 2 sections, looking at the evolving demographics of help-seeking gamblers (looking at a clinical population as opposed to general public targeted by the NCPG survey) over the years and the predictors of treatment outcomes. In the conclusion/ discussion section, the paper will do an exploration of the clinical implications of these findings.

\section{Discussion}

The demographics of help-seeking gamblers over the years (demographic differences)

In $2010^{3}$ and $2014,{ }^{4}$ NAMS conducted studies on the demographics of help-seeking gamblers from 2 separate cohorts (help-seekers from 2001-2008 and 2009-2012). The outcomes were largely similar; help-seekers were predominantly Chinese (95\% versus $90.1 \%$ ) males $(87.7 \%$ versus $89.4 \%$ ), with a mean age hovering around 40 (40 vs 39.6). There was however, a marked increase, observed in the percentages of help-seekers diagnosed with a co-occurring psychiatric disorder (e.g. another addictive disorder, a mood disorder, etc) between the older (2001-2008) and the more recent (2009-2012) cohort. These findings may have some possible (but not necessarily causal) implications, i.e.

a. More patients with co-morbidities are driven to use gambling as a way to cope with their issues,

b. The consequences of gambling has resulted in more patients getting into psychological distress,

c. Better and more stringent screening for co-morbidities among gamblers seeking help in NAMS or

d. A combination of (a), (b) and (c).

\section{Predictors of treatment outcome}

NAMS provides assessment and treatments (psychiatric/ medical and psychosocial) for gambling disorders and other behavioural addictions such as alcohol, drugs, gaming and internet. In terms of psychosocial treatment for gambling disorders, NAMS offers both individual and group therapy (for both patients and their family members) that are largely guided by an overarching framework that is guided by both cognitive behavioural therapy and motivational interviewing. 
In 2012, Guo et al. ${ }^{5}$ did a study examining demographic, clinical, behavioural and treatment program predictors of gambling frequency at 3,6 and 12-months, among PGs treated at an addiction clinic (NAMS) in Singapore using measures like the Hospital anxiety and depression scale, gambling symptom assessment scale (GSAS), personal wellbeing index (PWI), ${ }^{7}$ treatment perception questionnaire and gambling readiness to change scale. ${ }^{8}$ Treatment response in relation to changes in symptom severity, personal wellbeing and abstinence were also assessed. In the study, abstinence rates were found to be $38.6,46.0$ and $44.4 \%$ at 3, 6 and 12-months respectively. Significant reductions in gambling frequency, GSAS, and improvement in PWI were reported between baseline and subsequent outcome assessments, with the greatest change occurring in the initial three months. It was also found that being unemployed, having larger than average debts, poor treatment satisfaction and attending fewer sessions at the later stages of treatment were associated with significantly poorer outcomes, up to 1-year after initiating treatment. These findings show promise for the effectiveness of a CBT-based treatment approach for the treatment of Predominantly Chinese pathological gamblers.

In a more recent paper by Manning et al., ${ }^{9}$ it was reported that at 3-month follow-up, the quality of life in helping-seeking gambling patients from NAMS improved, $57.4 \%$ of them reported abstinence, and significant reductions were also observed in frequency and problem severity (all $\mathrm{P}<0.001$ ). It was also found that significant predictors of clinically meaningful improvement in frequency and problem severity included being a strategic gambler, exposure to gambling after 21 years of age, gambling on more days and selfreported problem seriousness at baseline, and higher treatment satisfaction.

In both the 2012 and 2014 study, treatment satisfaction seemed to be an important predictor of good outcomes, indicating the importance of constantly seeking patient feedback during clinical sessions and acting upon their feedback if they are deemed to be feasible and therapeutic for the patient. This, interestingly coincided with a study by Monnat, Bernhard, Abarbanel, John \& Kalina ${ }^{10}$ evaluating the relationship between treatment service quality, perceived improvement in social, functional, and material well-being and reduction in gambling behaviors among clients of Nevada statefunded pathological gambling treatment programs. The analyses revealed that client satisfaction with treatment services is positively associated with perceived improvements in social, functional, and material well-being, abstinence from gambling, reduction in gambling thoughts and reduction in problems associated with gambling,

What stands out in the 2014 study in terms of the predictors of good treatment outcome included a later exposure to gambling (after the age of 21) and being a strategic gambler. The former (later exposure to gambling) somewhat support the theory of how addiction can affect the development of the pre-frontal cortex (which makes early exposure detrimental) and how treatment can have a better impact for patients with a less impaired pre-frontal cortex. These findings are in line with a study by Rahman et al. ${ }^{11}$ indicating that earlier age of onset in gambling was associated with higher frequency of at-risk/problem gambling as opposed to later onset. The latter (strategic gamblers demonstrating better outcomes) seems to be linked to the type of psychological intervention (mainly CBT) which was administered to gamblers in treatment. In NAMS, CBT was the primary psychological intervention utilized for the treatment of disordered gamblers and CBT mainly targets on cognitive distortions that are related to one's chances of winning in gambling (e.g. illusion of control, lack of appreciating for the independence of events, misinterpretations of near misses, etc). These findings coincided with another study by Manning, Teoh, Guo, Wong \& Li, ${ }^{9}$ which suggested an absence in executive functioning deficits despite higher traits of impulsivity in the group of Asian gamblers seeking help from NAMS, indicating the suitability of interventions like CBT which emphasizes the use of self-monitoring and informed decision-making to reduce impulsive behaviours. In the same cognitive study, Manning, Teoh, Guo, Wong $\& \mathrm{Li}^{9}$ found greater levels of everyday cognitive failures, symptoms of dysexecutive syndrome and poorer executive functioning among "non-strategic" gamblers as compared to their "strategic" counterparts. It is helpful to note that "strategic" gamblers for the purpose of the study, refer to a group of gamblers to prefers to engage in types of gambling that seemingly appears to require some form of skill, technique, experience or calculation (e.g. sports betting, table games in the casino, horse-betting) while the "non-strategic" gamblers refer to those who are more likely to pick gambling activities that appear to rely more on chance or luck (e.g. lottery, slot machines)..$^{12}$ This is another important finding as it indicates in some ways that there might be some limitations in the current psychological treatment methods which do not seem to cater to the needs of "non-strategic" gamblers as well as to the "strategic" gamblers. It is therefore important to explore deeper into the psyche of this other group of gamblers who may be driven to gamble for a different set of reasons (e.g. to escape from distressing emotions or daily problems) altogether. With a better understanding of this group of "non-strategic" gamblers, the team can then look into more exclusive treatment pathways to better meet their needs.

\section{Clinical implications/ Recommendations}

\section{Importance of treatment and intervention}

In a study that was mentioned earlier in this paper, ${ }^{9}$ it was reported that the quality of life in helping-seeking gambling patients from NAMS improved, with $57.4 \%$ of them reporting abstinence and significant reductions (observed in frequency and problem severity) after being in treatment for 3 months. This clearly highlights the importance of being involved in some form of treatment and therapeutic intervention. One limitation, however, in this variable is the lack of a randomized control trial to compare the outcomes of gamblers who didn't seek treatment as opposed to this group of treatment-seeking gamblers.

\section{Gamblers with co-morbidities}

Firstly, the importance of screening for co-morbidities in gamblers and the exploration of a treatment pathway to help this group of gamblers address their co-occurring disorders. For example, the role of pharmacotherapy (e.g. prescription of Ritalin) and psychological treatment (e.g the infusion of Mindfulness based therapy) may come into play in such treatment pathways.

\section{Intensity of treatment based on the best treatment outcomes with the first 3 months of treatment}

The findings also suggested that early treatment satisfaction is paramount in improving short-term outcomes, with baseline gambling behaviour and treatment intensity playing a more significant role in the longer term. As it was also indicated that the greatest positive changes in these gamblers seeking treatment occurs in the initial three months, 
it may imply that a treatment approach that engages the patients at a higher level of intensity in the earlier stages followed by an eventual tailing down of sessions may be a more efficacious way of getting the "maximum bang for the buck" in terms of treatment outcomes.

This is an important piece of finding that has influenced NAMS in its guidelines for the implementation of psychological treatment for gamblers where the patient is seen and engaged most intensely in the first 4 to 6 weeks (preferably weekly), after which sessions will be tailed down to once every fortnightly and subsequently monthly when the patient has demonstrated stability in terms of his/her treatment outcomes.

\section{Better treatment prognosis for late exposure to gambling}

The other finding which may have more preventive rather than clinical implications is the indication of better treatment outcomes for gamblers who started getting exposed to gambling after the age of 21. This piece of information may be helpful in terms of educating parents/educators/general public about the harms of early exposure to gambling and regulations that protects the youth from being exposed to gambling at too early an age. It may also be helpful to find out more about the types of treatment that may be efficacious for gamblers who were exposed early and if there is also a need for a separate treatment pathway that suits that needs better.

\section{Current CBT-based treatment approach efficacious for strategic gamblers}

The findings have also indicated the efficacy of NAMS's current psychological treatment strategies for disordered gamblers (primarily CBT-based) for strategic gamblers as their executive functioning is mostly well-preserved. This converges with the meta-analytical reviews of Gooding \& Tarrier ${ }^{13}$ and Pallesen, Mitsem, Kvale, Johnsen \& Molde ${ }^{14}$ indicating that CBT has the most empirical evidence for the treatment of pathological gambling. However, there is also a need to look at a separate treatment pathways for "non-strategic" gamblers who may be driven by other factors to gamble. Current psychological treatment approaches may require some integration with other treatment methods that can access other domains of psychological distress (e.g. emotional and physiological sensation domains).

\section{Conclusion}

Taken in all, these findings do have important clinical implications that would continue to guide the treatment philosophy and direction of NAMS. It is important to note that psychosocial treatment for gamblers in NAMS is mainly guided by a cognitive behavioural approach which is very much based on the original psychosocial treatment designed for treating patients with substance and alcohol use disorders. Looking ahead, there is a need to further examine the idiosyncrasies of disordered gambler so as to make treatment less generalized and more targeted to meet their recovery needs. It is also important to note that the participants involved in most of the studies discussed in this paper are samples of convenience where predictors of what seemed to work well in treatment are mainly derived via correlations and simple comparison of means and percentages. It may be helpful and more ground-breaking for future studies to look at new areas like a randomized controlled trial comparing groups of gambling patients assigned to different types of interventions to ascertain which particular intervention (e.g. groups versus individual, mindfulness based therapy versus CBT) works more efficaciously for different groups of gamblers. These ideas originates from a persuasive body of evidence provided by Ladouceur et al..$^{15} \&$ Zablocki ${ }^{16}$ lending support for the efficacy of group work in and Toneatto, Vettese \& Nguyen ${ }^{17}$ on the role of mindfulness based therapy in the treatment of disordered gambling. Other forms of studies may also be conducted at some point to look at Predictors of treatment seeking behaviour in gamblers, ${ }^{18}$ Barriers to Seeking Help for Gambling Problems, ${ }^{19}$ Outcome of Pharmacological Treatments of Pathological Gambling ${ }^{20}$ or useful components (e.g distorted cognitions or negative affect) to focus in treatment. ${ }^{21}$

\section{Acknowledgements}

Victoria Manning

Wong Kim Eng

\section{Conflict of interest}

The author declares no conflict of interest.

\section{References}

1. Man called girlfriend before setting himself and car on fire. AsiaOne, Singapore; 2014

2. Casinos in Singapore will create havoc. Topix0 LLC, Singapore; 2014.

3. Lee KM, Guo S, Manning V, et al. Demographic and Clinical Features of 350 Pathological Gamblers in Treatment. Poster presented at the Asia Pacific Behavioral and Addiction Medicine Conference, Singapore; 2010 .

4. Ng A, Koh Pk, Ong R, et al. Predictors of treatment dropout: A survival analysis of pathological gambling patients treated in a Singapore addictions clinic. Poster/Oral presentation at the Singapore Health \& Biomedical Congress, Bronze award, Singapore; 2014.

5. Guo S, Manning V, Thane KK, et al. Predictors of Treatment Outcome Among Asian Pathological Gamblers (PGs): Clinical, Behavioural, Demographic, and Treatment Process Factors. J Gambl Stud. 2012;30(1):89-103.

6. Kim SW, Grant JE, Potenza MN, et al. The Gambling Symptom Assessment Scale (G-SAS): A reliability and validity study. Psychiatry Res. 2009;166(1):76-84.

7. Tomyn AJ, Tyszkiewicz MD, Cummins RA. The Personal Wellbeing Index: Psychometric Equivalence for Adults and School Children. Soc Indic Res. 2011;110(3):913-924.

8. Neighbors C, Lostutter TW, Larimer ME, et al. Measuring Gambling Outcomes Among College Students. J Gambl Stud. 2002;18(4):339-360.

9. Manning V, Teoh HC, Guo S, et al. Executive functioning in Asian pathological gamblers. International Gambling Studies. 2013;13(3):403-416.

10. Monnat SM, Bernhard B, Abarbanel BL, et al. Exploring the Relationship Between Treatment Satisfaction, Perceived Improvements in Functioning and Well-Being and Gambling Harm Reduction Among Clients of Pathological Gambling Treatment Programs. Community Ment Health J. 2013;50(6):688-696

11. Rahman AS, Pilver CE, Desai RA, et al. The relationship between age of gambling onset and adolescent problematic gambling severity. $J$ Psychiatr Res. 2012;46(5):675-683.

12. Odlaug BL, Marsh PJ, Kim SW, et al. Strategic vs nonstrategic gambling: Characteristics of pathological gamblers based on gambling preference. Ann Clin Psychiatry. 2011;23(2):105-112. 
13. Gooding P, Tarrier N. A systematic review and meta-analysis of cognitive behavioural interventions to reduce problem gambling: hedging our bets? Behav Res Ther. 2009;47(7):592-607.

14. Pallesen S, Mitsem M, Kvale G, et al. Outcome of psychological treatments of pathological gambling: a review and metaanalysis. Addiction. 2005;100(10):1412-1422.

15. Ladouceur R, Sylvain C, Boutin C, et al. Group therapy for pathological gamblers: a cognitive approach. Behav Res Ther. 2003;41(5):587-596.

16. Elaine Zablocki. Group Therapy Can Help Pathological Gamblers. Clinical Psychiatry News. 2006;34(8):46 p.

17. Tony Toneatto, Lisa Vettese, Linda Nguyen. The role of mindfulness in the cognitive-behavioural treatment of problem gambling. Journal of Gambling Issues. 2007;19:91-100.
18. Valdivia Salas S, Blanchard KS, Lombas AS, et al. Treatment-seeking precipitators in problem gambling: Analysis of data from a gambling helpline. Psychol Addict Behav. 2014;28(1):300-306.

19. Suurvali H, Cordingley J, Hodgins DC, et al. Barriers to Seeking Help for Gambling Problems: A Review of the Empirical Literature. $J$ Gambl Stud. 2009;25(3):407-424.

20. Pallesen S, Molde H, Arnestad HM, et al. Outcome of Pharmacological Treatments of Pathological Gambling. J Clin Psychopharmacol. 2007;27(4):357-364.

21. Fortune EE, Goodie AS. Cognitive distortions as a component and treatment focus of pathological gambling: A review. Psychol Addict Behav. 2012;26(2):298-310. 